\title{
Experimental Study on the Hygrothermal Performance of Zeolite-Based Humidity Control Building Materials
}

\author{
Bo Zhou ${ }^{1,2}$ and Zhenqian Chen ${ }^{1 *}$ \\ ${ }^{1}$ IIUSE, School of Energy and Environment, Southeast University, Nanjing 210096, P. R. China \\ ${ }^{2}$ SUST, School of Environment Science and Engineering, Suzhou University of Science and \\ Technology, Suzhou215009, P. R. China
}

Email: zqchen@seu.edu.cn

\begin{abstract}
The hygrothermal performances of three types of zeolite-based humidity control building materials (ZBHCMs) were experimentally studied. The distributions of the pores and matrix of the ZBHCMs were visually characterized by micrographs. The saturated solution method was applied to measure the humidity control performance of the ZBHCMs. The effects of porosity, ambient temperature and relative humidity (RH) on the humidity control performance of the ZBHCMs were studied. The experimental results indicated that the humidity control performance of ZBHCMs is strongly affected by the porosity and the pore diameter. The environmental temperature and the RH have considerable influence on the adsorption performance of ZBHCMs. The desorption performance of ZBHCMs is affected more strongly by the ambient RH.
\end{abstract}

Keywords: Zeolite-based humidity control building material, Adsorption, Desorption, Pore structure.

\section{INTRODUCTION}

Humidity is very important to human health, and living environment, such as relative humidity indoors being too high or too low and thus having an adverse effect on our living conditions [1-4]. Additionally, an excessively high or low relative humidity $(\mathrm{RH})$ of the indoor air can cause various problems in the lives of people, such as the comfort of the occupants, the storage of goods, the durability of the outer structure of the building and energy consumption [510]. When indoor relative humidity is maintained at $30 \%$ $60 \%$, the body feels most comfortable [11-13]. The heating, ventilating and air conditioning system is typically used to control the $\mathrm{RH}$ of indoor air and is a primary component of building energy consumption. A humidity control material (HCM) can adsorb or release moisture automatically without any power source or mechanical equipment due to its sensitivity to the variations of ambient temperature and $\mathrm{RH}$ [14-16]. Thus, the use of a HCM is of great importance to the indoor environment, energy conservation and sustainable development of the ecological environment.

Since the use of a HCM was initially proposed by a Japanese scholar [17], it has received considerable interest globally. Various HCMs were developed, including biomass HCM [18-19], inorganic HCM [20-24], and organic HCM [25-26]. Horikawa [27] studied the humidity control capacity of activated carbon from bamboo. The optimal treatment for the highest humidity control capacity was obtained. Wang
[28] prepared an acrylate-based copolymer emulsion and investigated the humidity controlling characteristics in an interior wall coating.

The research studies described above experimentally characterized the HCM on the macroscopic scale, primarily examining the original material selection, the optimal treatment and the best mixed portions of raw materials, as well as the humidity control performance measurement of the HCM. As a typical porous media, the micro-structure of HCM might have an influence on the humidity control performance of the HCM [30-33]. To the best of our knowledge, there are only a small number of reports describing research in this area. Tomita [34] investigated the humidity control ability of monolithic bimodal porous silica gel. The humidity control performance of the gel was controlled by changing its mesopore size. The presence of macrospores enabled the gel to quickly respond to a change in humidity, due to the rapid gas diffusion in the monolithic gel body. Watanabe [35] investigated the humidity control abilities of allophone, silica gel and gibbsite, which had mesopores. The results indicated that the mixture of gibbsite and clay minerals is appropriate for use as interior wall building materials, and these materials exhibit superior performance in the removal of formaldehyde.

The above studies analyzed the humidity control abilities of HCM from the perspective of pore size. The results indicated that the humidity control performance of HCM varies with mesopore size. However, detailed micro-pore 
structure parameters which affected the heat or moisture migration in HCM must be further studied. Therefore, this work will prepare ZBHCMs by mixing mineral zeolite, cement, pulverized fuel ash, poplar cellulosing, and mildew preventive and antimicrobial agents. The effects of the micropore structure on the heat and moisture migration in ZBHCMs will be studied."

\section{PREPARATIONS OF ZBHCMS}

\subsection{Optimization of the zeolite performance by calcinations}

Zeolite, a siliceous sedimentary rock abundant all over the world, is receiving significant attention due to its unique combination of physical and chemical properties. Figure. 1 shows micrographs of raw and calcinated zeolite taken using a scanning electron microscope (SEM). As shown in Figure. 1(a), the raw zeolite is observed to have a round plate structure with loose pore distribution. Figure 1(b) shows the micro-pore morphology of zeolite changes after performing calcinations at $500{ }^{\circ} \mathrm{C}$ for 3 hours. The pores become smaller, and the number of small pores increases. As shown in Figure 1(c) and Figure 1(d), the energy spectra of impurity elements (Fe, Al) almost disappeared after calcinations at $500{ }^{\circ} \mathrm{C}$ for three hours, which implies that the purity of zeolite increases after calcinations.

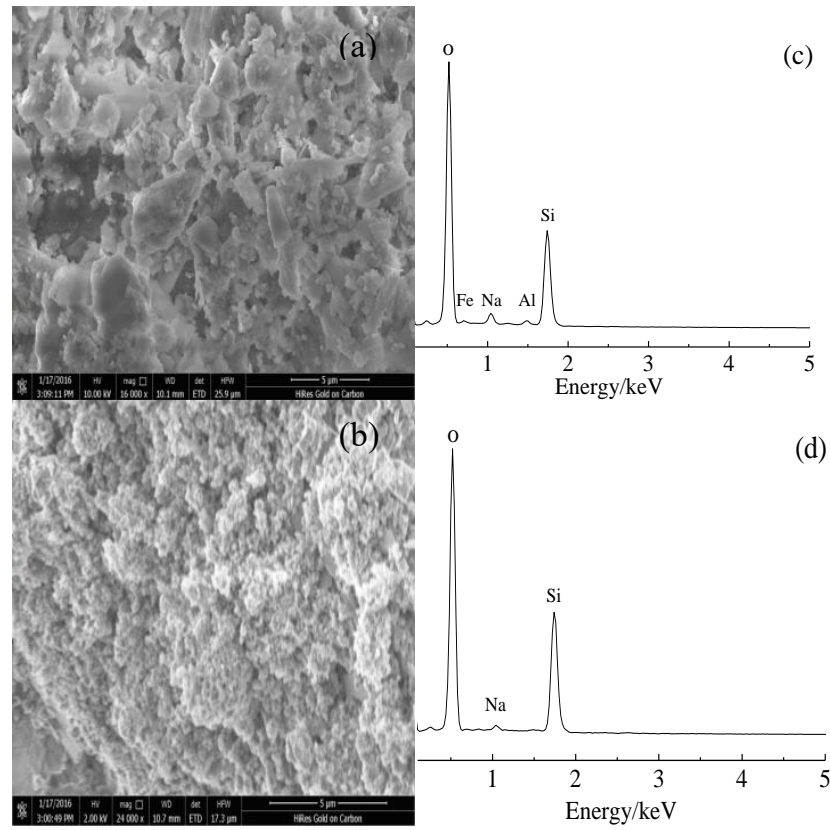

Figure 1. SEM and EDS of raw and calcinated zeolites

Zeolite that exhibits small and dense pores after calcination can improve the humidity control performance of zeolite. A comparison of the adsorption and desorption performances between raw zeolite and calcinated zeolite are shown in Figure. 2. It is evident that the adsorption content in calcinated zeolite is higher than that in the raw zeolite, which shows that calcinated zeolite has a superior ability to regulate and control the indoor RH for buildings. This ability is attributed to the fact that small and dense pores can provide higher capillary forces. Meanwhile, calcinated zeolite exhibits a slower speed in releasing excess moisture because small and dense pores promote water retention. The slow and uniform desorption speed can create a more comfortable indoor environment for people. Therefore, calcination can improve the adsorption and desorption performances of zeolite.

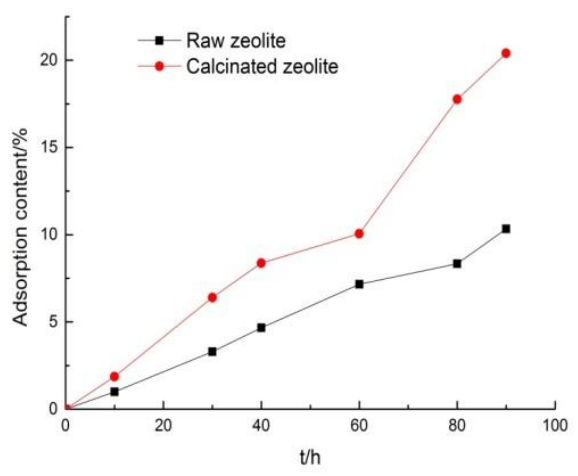

(a) Adsorption

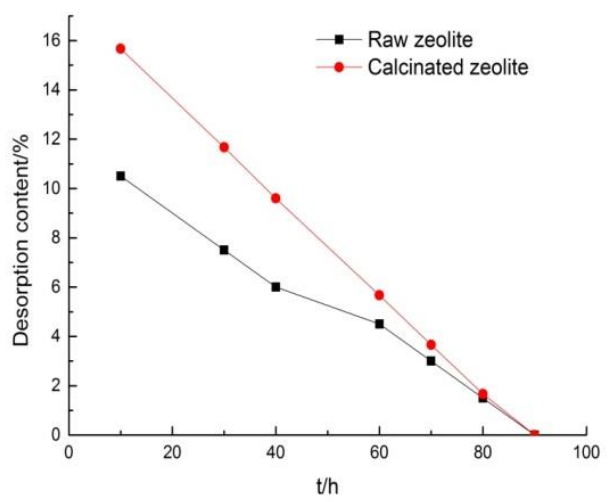

(b) Desorption

Figure 2. Adsorption and desorption contents of raw and calcinated zeolites

\subsection{Preparation of $\mathrm{ZBHCMs}$}

When preparing ZBHCMs, several raw materials were mixed at a certain ratio. Natural mineral zeolite was used as the main moisture modulating ingredient. It was important that the calcinations of zeolite should be performed at a certain temperature for a specific time to increase the porosity and pores connectivity of the ZBHCMs. Cement was applied for structural support. Pulverized fuel ash, an industrial waste from coal combustion, was used as a supplement in the composite. Because pulverized fuel ash is a product pollutant, using pulverized fuel can not only provide a way to utilize the waste and promote the sustainable development of ecological environment but also reduce the cement and water consumption, thereby lowering the cost of building materials. Poplar cellulosine was used as a suplemntary humidity control material. The well-developed fibrous microcellular structure of poplar cellulosine was able to maintain balance between the adsorption and desorption performances of ZBHCMs. Mildew preventive and antimicrobial substances were added to ZBHCMs for preservation. All of the abovementioned components were mixed, pulped and poured into molds. After the composites were shaped, the tested blocks were removed from the molds and maintained at a certain temperature and $\mathrm{RH}$ to achieve the best strength. 


\section{STRUCTURAL CHARACTERIZATIONS ZBHCBMS}

OF

The effects of micro pore structures (physical factor) on humidity control performance of ZBHCBMs were investigated. It was found that pore size distribution is directly relevant to capillary force of ZBHCBMs, while surface free energy directly affects the interaction force between water vapor in air and ZBHCBMs. So, the pore structures have a significant effect on humidity control performance of ZBHCBMs.

To elucidate the effect of the micro-structure of ZBHCBMs on its humidity control performance, micrographs and binary images of ZBHCBMs of different porosity levels in Figure 3 were obtained using microscope and image analysis methods. The proportion of the raw materials and the stirring speed were the same when preparing the blocks $\# 1, \# 2$, and \#3, while the stirring times for the three blocks were $5 \mathrm{~min}, 10 \mathrm{~min}$, and $15 \mathrm{~min}$, respectively. Therefore, the micro-structure of each of the blocks was different. As shown in Figure. 3, the black portion represents the pores and the white portion represents the framework. The porosity level of the blocks decreases with the increasing of stirring time, as these raw materials become better mixed. The corresponding pore distributions were characterized using the statistical analysis method shown in Figure. 4. The pore diameter of the ZBHCBMs was found to become more uniform with an increase in stirring time.

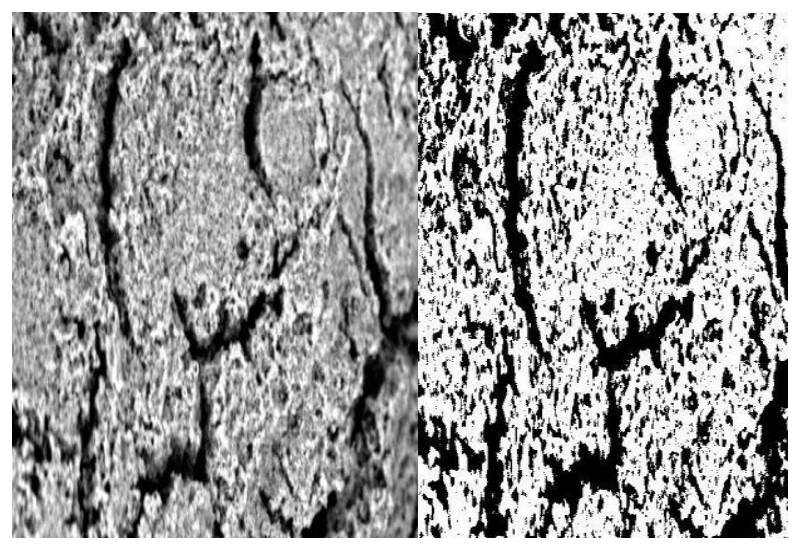

(a) $\# 1$ (porosity $=0.83)$

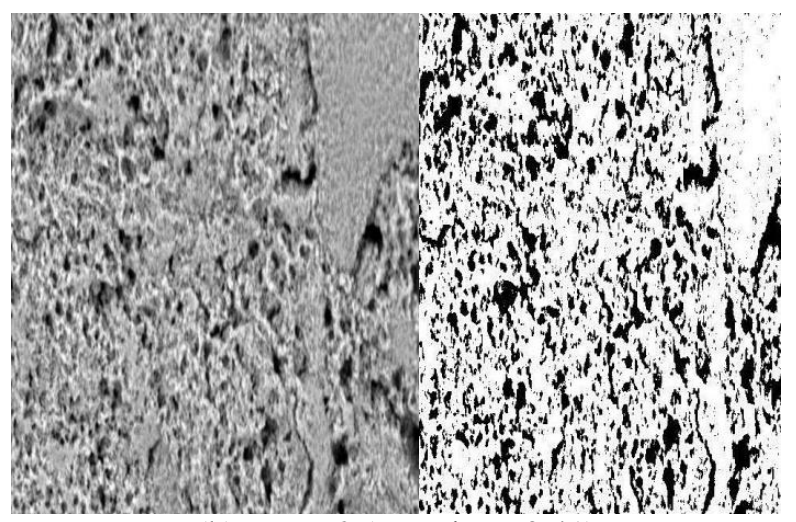

(b) $\# 2($ porosity $=0.41)$

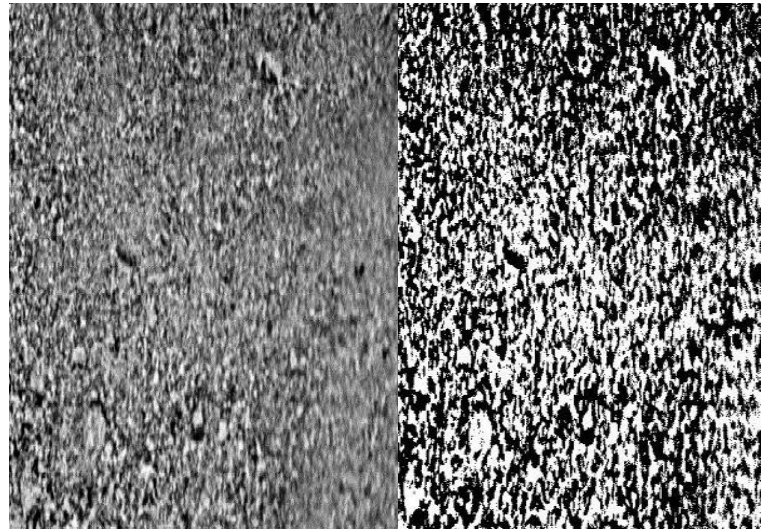

(c)

$\# 3($ porosity $=0.14)$

Figure 3. Micrograph and binary image of the ZBHCBMs

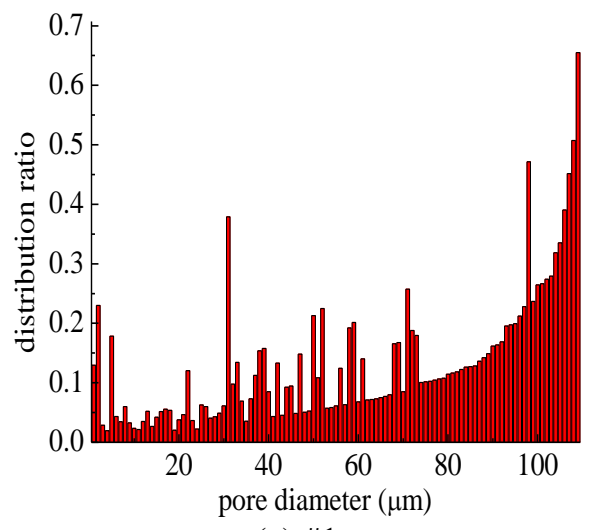

(a) \#1

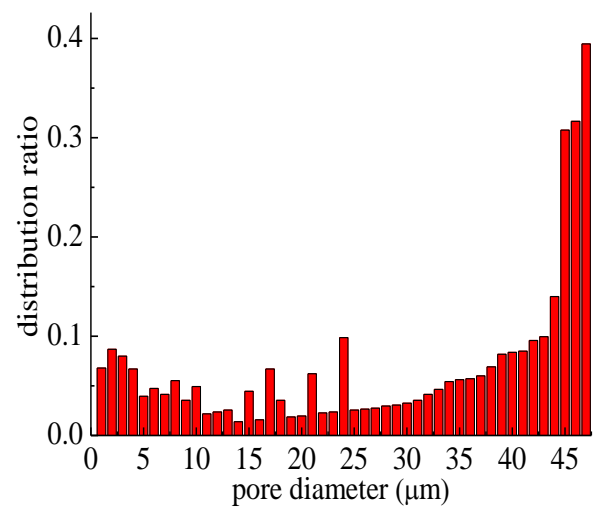

(b) \#2

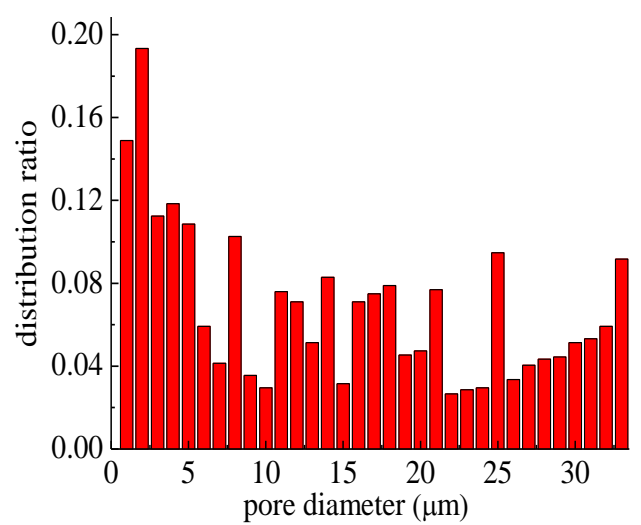

(c) \#3

Figure 4. Pore distribution diagrams of the ZBHCBMs 


\section{SURFACE FREE ENERGY}

Surface free energy is influenced by the surface temperature, the surface shape and curvature, the surface dispersion force and the dipolar force. Therefore, the capabilities of adsorbing or desorbing moisture from ambient air are different among the different materials or the different parts of a material. The contact angles of the two blocks were measured by an optical contact angle measuring device, as shown in Figure. 5. A sample with a large porosity level exhibits a small contact angle for water. A sample with a small contact angle for water has a high surface free energy, resulting in a strong capability for ZBHCBMs to adsorb liquid water and a weak ability to absorb water.

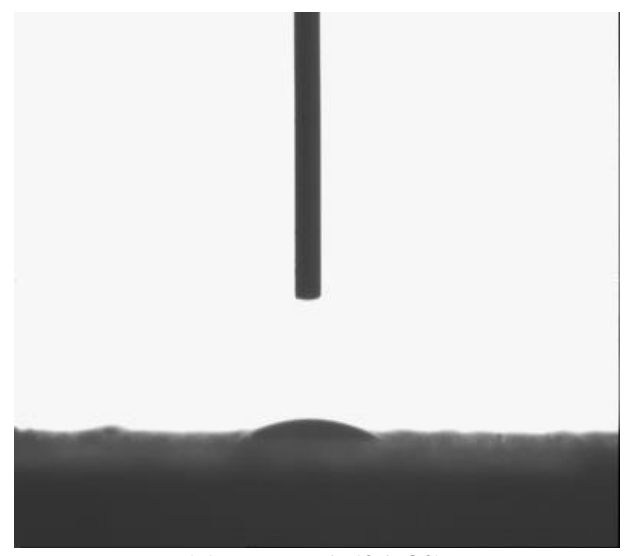

(a) $\# 1\left(21.3^{\circ}\right)$

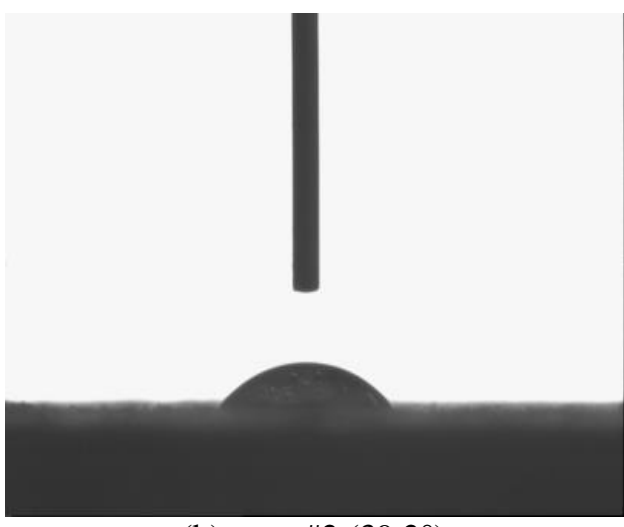

(b)

$\# 2\left(39.2^{\circ}\right)$

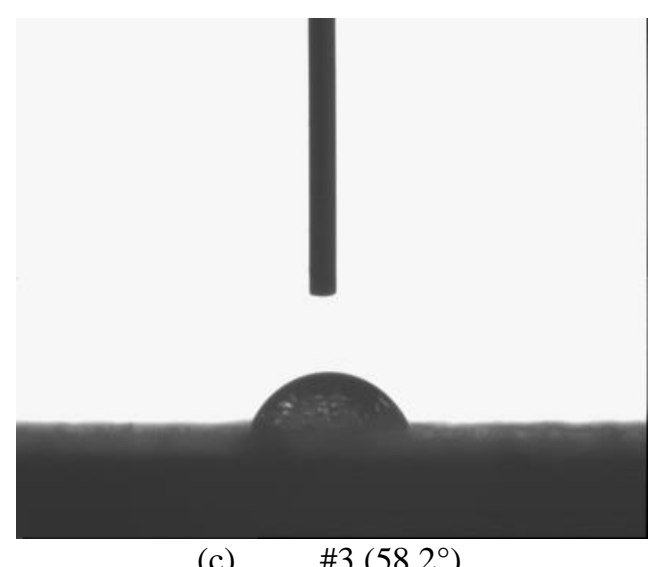

(c)

$\# 3\left(58.2^{\circ}\right)$

Figure 5. Surface contact angle of the ZBHCBMs

\section{HUMIDITY CONTROL MEASUREMENTS OF ZBHCMS}

PERFORMANCE

\section{1 Experimental apparatus}

The schematic diagram of the experimental apparatus used in the test is shown in Figure.6. The apparatus consisted of three systems: test chamber system, data acquisition system and blocks weighting system. The test chamber system consisted of a climatic chamber, testing blocks, and riser vent. The data acquisition system was comprised of a T\&RH sensor (EE31-A), the data acquisition equipment and a recording computer. The T\&RH sensor (with an accuracy of temperature of $\pm 1{ }^{\circ} \mathrm{C}$ and an accuracy of $\mathrm{RH}$ of $\pm 2 \%$ ) was positioned above the blocks to monitor the temperature and humidity of the chamber. All the temperature and humidity data were recorded using an Agilent 34970A data acquisition system, which was connected to the recording computer. The weighing system for the blocks was a high precision electronic balance FA2004N with an accuracy of $0.0001 \mathrm{~g}$.

The experimental setup was used to maintain the experimental temperature and $\mathrm{RH}$ within a certain range and to record the variations of the adsorption and desorption contents of the ZBHCMs at certain times.

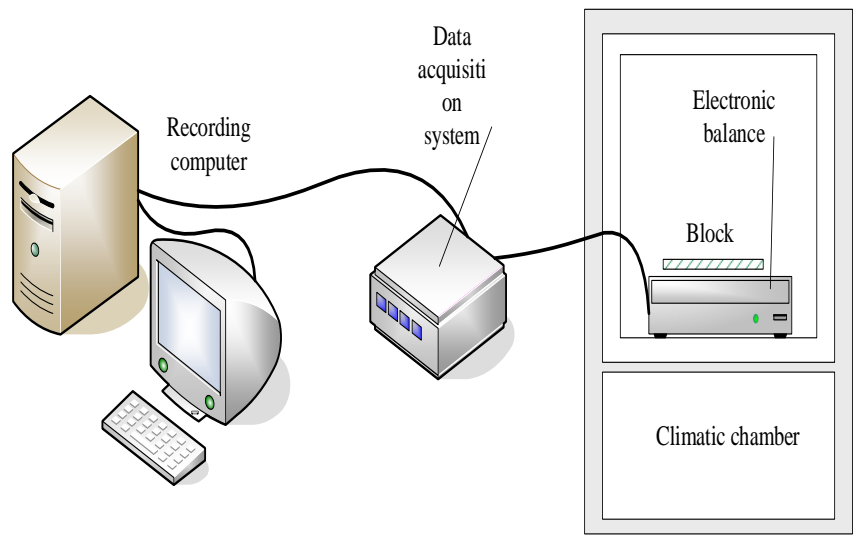

Figure 6. Schematic diagram of the experimental apparatus

\subsection{Measurement of humidity controlling functions of zeolite}

In order to measure the humidity controlling properties of the modified zeolite samples, increasing humidity and dehumidifying properties were measured in both dry and moist environments. Firstly, the zeolite samples were placed in glass plates or beakers, and dried. When the weight changes in the samples were less than $1 \%$ for approximately 12 hours, the blocks were considered and dried. Next, the testing samples were moved into a desiccator. The functions of these zeolite samples were measured in an artificial climate box as follows:

(a) Dehumidifying properties: In an artificial climate box, the relative humidity (RH) was adjusted to below $30 \%$ at $25^{\circ} \mathrm{C}$. Zeolite samples, previously saturated with water, were placed in the artificial climate box. During the first six hours, the testing samples were measured once every hour. Next, the samples were measured two times a day until the variations of their weights were within $1 \%$ for approximately 240 hours. The curve of RH vs. time was recorded. 
(b) Increasing humidity properties: In an artificial climate box, the RH was increased to more than $85 \%$ at $25^{\circ} \mathrm{C}$. After the drying process, Zeolite samples were placed in the artificial climate box. The testing samples were measured according to the same time schedule as that of the desorption experiment. However, the time when the variations of their weights were within $1 \%$ was approximately 180 hours. The curve of RH vs. time was recorded.

\subsection{Testing protocol of the $\mathrm{ZBHCMs}$}

After the blocks were fabricated (as described in Part 2), they were placed into a drying oven $\left(120^{\circ} \mathrm{C}\right)$ to remove the moisture. When the weight changes of the blocks were less than $1 \%$ for approximately 12 hours, the blocks were considered as well dried. Next, the testing blocks were moved into a desiccators. After the temperature of the blocks reached ambient temperature, they were characterized in the adsorption experiment. It is important to perform waterproof treatment on the side and bottom surfaces other than the adsorption / desorption surface to model a one-dimensional heat and moisture migration environment. During the experiment, the temperature and $\mathrm{RH}$ of the test chamber were regulated to the required values. The initial weights of the testing blocks were measured before they were placed into the artificial environment. In the first six hours, the testing blocks were measured every hour. Next, the blocks were measured two times a day until the variations of their weights were within $1 \%$ for approximately 240 hours. In the desorption experiment, the saturated adsorption testing blocks were moved into the pre-set relatively low humidity environment. The testing blocks were measured according to the same time schedule as that of the adsorption experiment. However, the time when the variations of their weights were within $1 \%$ was approximately 180 hours.

The adsorption or desorption content of the ZBHCMs was calculated using the following formula:

$C=\frac{W-W_{0}}{W_{0}} \times 100 \%$

where $W$ is the moisture adsorption or desorption weight of the ZBHCM, and $W_{0}$ is the initial weight of the ZBHCM.

\subsection{Characteristic values and properties of ZBHCM}

Most Equilibrium isothermal moisture absorption relation of building material can be expressed as follows:

$$
C=a \phi^{b}+c \phi^{d}
$$

where, $\phi$ is ambient relative humidity, a, b, c, d are fitting coefficients.

The experimental facility is shown in Figure. 7, which consisted of a HS-150L constant temperature humidity chamber, high precision electronic balance FA2004N with an accuracy of $0.0001 \mathrm{~g}$. The temperature was set as $20{ }^{\circ} \mathrm{C}$ 。 $\mathrm{ZBHCM}$ was placed into a drying oven $\left(120{ }^{\circ} \mathrm{C}\right)$ to remove the moisture. When the weight changes of ZBHCM was less than $1 \%$, it was moved into a desiccator. After the temperature of the blocks reached ambient temperature, measuring the moisture content variation of ZBHCM can started when the ambient relative humidity are $33 \%$ and $85 \%$. Finally, the characteristic values and properties of ZBHCM were obtained by fitting these experimental data which is shown in Figure. 8.

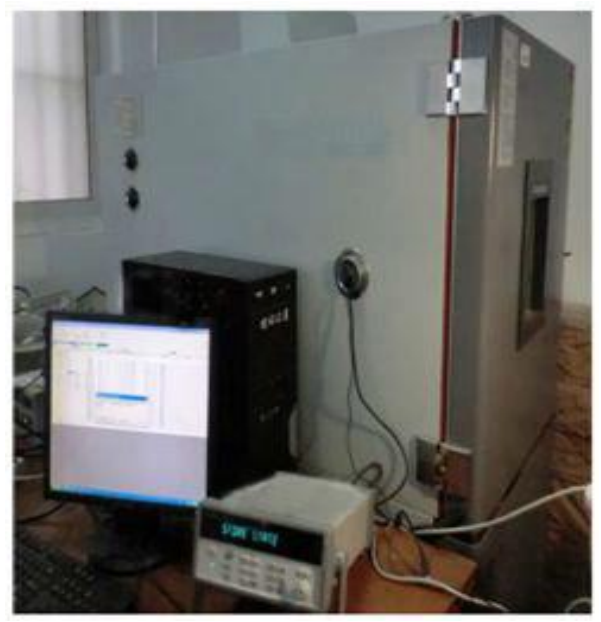

Figure 7. Moisture absorption test facility

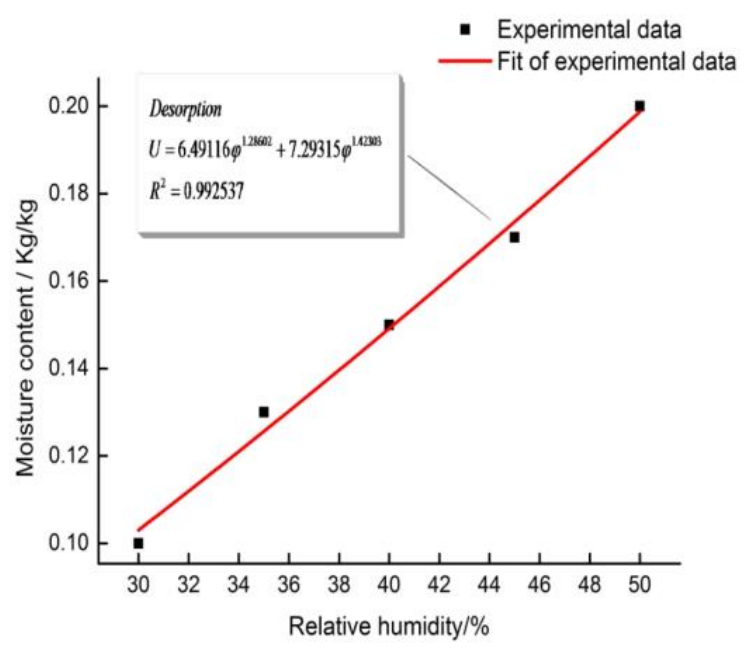

(a) Adsorption

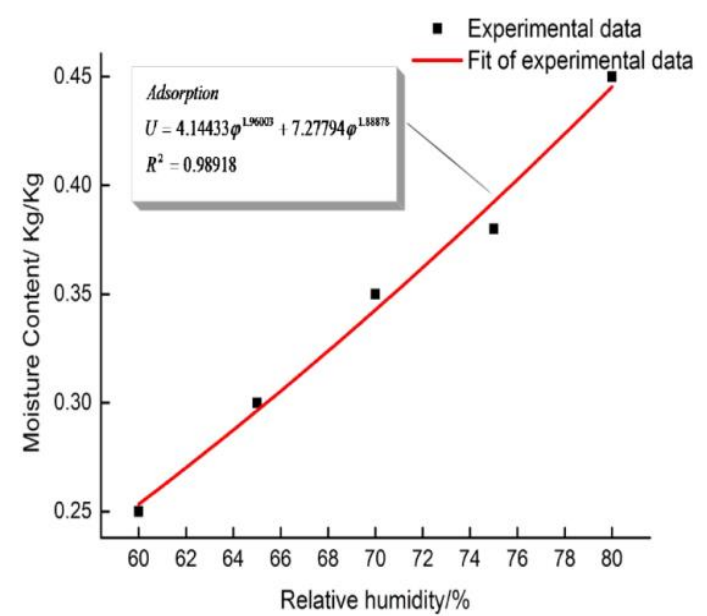

(b) Desorption

Figure 8. Hygroscopicity Figureure 


\section{EXPERIMENTAL RESULTS AND DISCUSSION}

\subsection{Effects of the pore structure and the surface free energy on the humidity control properties}

Figure. 9 illustrated the adsorption and desorption contents of the ZBHCMs with different porosity levels. The temperature and $\mathrm{RH}$ of the adsorption process in the test chamber were set as $20{ }^{\circ} \mathrm{C}$ and $85 \%$, respectively, while the temperature and $\mathrm{RH}$ of the desorption process in the test chamber were set as $20{ }^{\circ} \mathrm{C}$ and $33 \%$, respectively. As shown in Figure 9, the adsorption speed curves in the first six hours exhibit sharp slopes due to the high concentration gradient of the moisture between the chamber environment and within the testing blocks. The adsorption speeds decrease with the increasing of time in the testing blocks. The ZBHCMs of lower porosity levels were observed to be able to adsorb more moisture compared with the other two types of ZBHCMs. The adsorption content of \#3 (porosity $=0.14$ ) is approximately two times higher than that of \#1 (porosity = 0.83 ). During the desorption process, the rate of desorption decreases with an increase of time in the testing blocks. However, a higher amount of moisture remains in the testing blocks of relatively small porosity levels (\#2, \#3). The ZBHCM with small porosity and tiny pore diameter exhibits a small surface contact angle (large surface free energy), which can exhibit a large capillary force, thereby enabling water retention in the testing blocks. Hence, when designing ZBHCMs, there is a reasonable pore size for superior humidity control performance with reduced water retention.

\subsection{Effect of the ambient temperature on the humidity control properties}

Figure. 10 shows the effect of the chamber temperature on the humidity control performance of a ZBHCM. The porosity of the testing block is 0.21 (\#2). The RH of the adsorption process in the test chamber was set to $85 \%$, while the RH of the process of desorption in the test chamber was set to $33 \%$. The trend of adsorption / desorption speed is similar to that in Figure 9. The humidity control performance increases with an increase in ambient temperature, decreases with a decrease in ambient temperature. As the chamber temperature increases, the saturated water vapor concentration in air increases, thereby causing the adsorption content of ZBHCM to increase; that is, the increase in the moisture concentration gradient between the chamber environment and within the testing block. Adsorption is a process of the condensation of water vapor in air which will release the condensation heat. However, chamber temperature has little influence on the desorption performance of the ZBHCM because of the pore capillary effect of ZBHCM. Kinetic energy of water vapor molecules adsorbed in ZBHCM increases in a low humidity environment with an increase in ambient temperature, so the ability to eliminate the water vapor intermolecular constraint increases. Moreover, desorption is a gasification process for water vapor to struggle to be released from ZBHCM which absorbs the ambient heat.

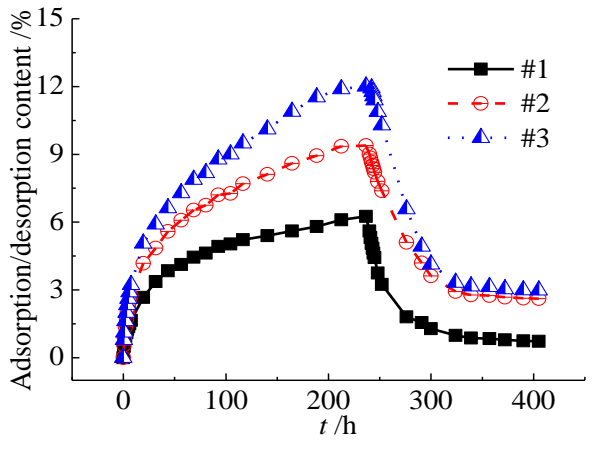

Figure 9. The effect of porosity on the humidity control performance of the ZBHCMs

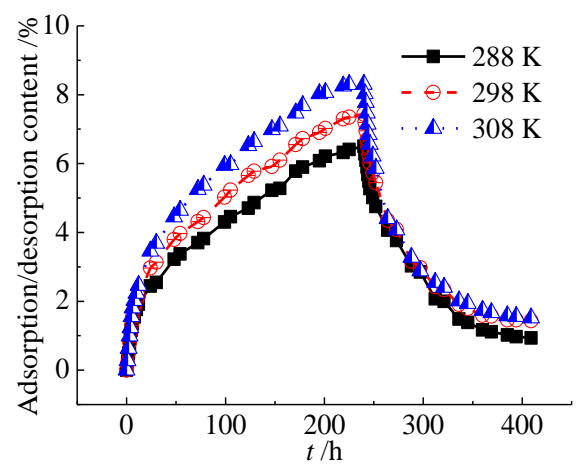

Figure 10. The effect of ambient temperature on the humidity control performance of the ZBHCMs

\subsection{Effect of the ambient RH on humidity control properties}

Figure 11 presents the effect of ambient $\mathrm{RH}$ on the humidity control properties of a ZBHCM. The porosity of the testing block is $0.11(\# 3)$. The temperature of the adsorption and desorption processes in the test chamber were set to 20 ${ }^{\circ} \mathrm{C}$. The maximum adsorption content was approximately $12 \%$ of its own weight when the $\mathrm{RH}$ was $85 \%$, and the minimum adsorption content was approximately $7.5 \%$ of its own weight when the RH was $33 \%$. The maximum desorption content was approximately $9 \%$ of its own weight when the RH was $85 \%$, and the minimum desorption content was approximately $6.5 \%$ of its own weight when the RH is $33 \%$. They are all caused by the RH gradients inside ZBHCMs and in air. A larger RH implies more moisture in the chamber environment. Therefore, a higher concentration gradient of moisture between the chamber environment and within the testing block can promote larger adsorption/desorption content in the testing block. The time that the adsorption becomes stable is always longer than the time it takes for the desorption to become stable, which indicates that the ZBHCM retains a certain amount of water or water vapor. Therefore, ZBHCMs must be treated to prevent the growth of bacteria and mildew. The adsorption content is always more than the desorption content, which agrees with the pore capillary effect of ZBHCM. 


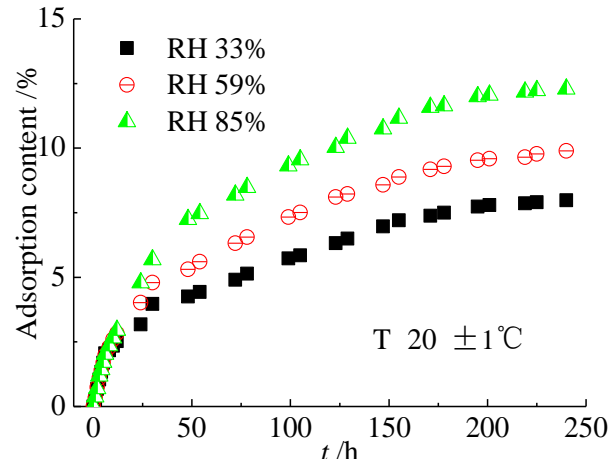

(a) Adsorption

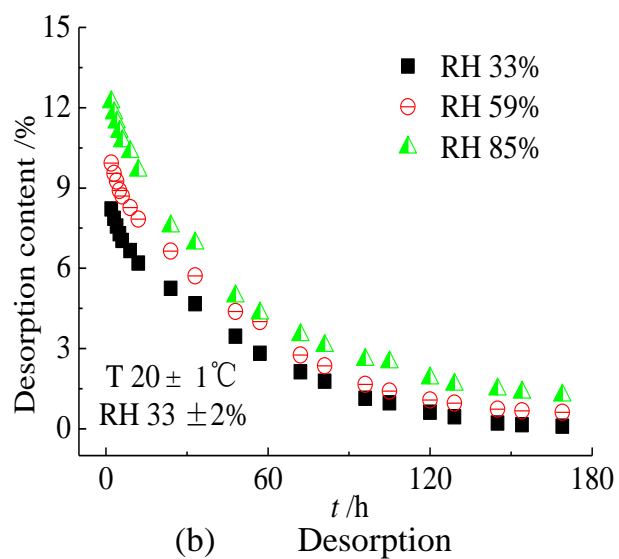

Figure 11. The effect of ambient $\mathrm{RH}$ on the humidity control performance of the $\mathrm{ZBHCMs}$

\section{CONCLUSIONS}

The experiments were performed investigating the hydrothermal performance of ZBHCMs. The effects of porosity, ambient temperature and $\mathrm{RH}$ on the humidity control performance of ZBHCMs were studied. The results indicated that the adsorption performance of a ZBHCM is strongly affected by the environmental temperature and $\mathrm{RH}$. The desorption performance of a ZBHCM is more affected by the ambient $\mathrm{RH}$, rather than the temperature. In addition, the micro-structure of a ZBHCM has a considerable influence on its humidity control performance. The ZBHCMs with small porosity and tiny pore diameter has higher adsorption capability as well as greater water retention in the blocks. Therefore, there is a reasonable pore size for superior humidity control performance with reduced water retention.

\section{ACKNOWLEDGMENT}

This work was financially supported by National Natural Science Foundation of China (No.51276041) and the Program of International Science and Technology Cooperation (No.2011DFA60290).

\section{REFERENCES}

[1] Raoudha Chaabane, Faouzi Askri and Sassi Ben Nasrallah. "Numerical analysis of heat transfer induced by a horizontal ground heat exchanger in a heterogeneous soil," International Journal of Heat and
Technology, vol. 28, no. 2, pp. 51-57, 2010.

[2] Zhi Yang and Min Quan Feng, "Hei River flood risk analysis based on coupling hydrodynamic simulation of 1-D and 2-D simulations," International Journal of Heat and Technology, vol. 33, no. 1, pp. 47-54, 2015. DOI: 10.18280/ijht.330107.

[3] M. Yun, B. Yu and J. Cai, "A fractal model for the starting pressure gradient for Bingham fluids in porous media," Heat Mass Transf, vol. 51, no. 5, pp. 14021408, 2008.

[4] L. Z. Zhang, "A fractal model for gas permeation through porous membranes," Heat Mass Transf, vol. 51, no. 21, pp. 5288-5295, 2008.

[5] H. S. Kang, D. Dennis and C. Meneveau, "Flow over fractals: drag forces and near wakes," Fractals, vol. 19 no. 04, pp. 387-399, 2011.

[6] R. Gomes-Fernandes, B. Ganapathisubramani and J. C. Vassilicos, "Particle image velocimetry study of fractal-generated turbulence," Fluid Mech., vol. 711, no. 2012 , pp. 306-336. DOI: 10.1017/jfm.2012.394.

[7] C. Zhang, Z. Deng and Y. Chen, "Temperature jump at rough gas-solid interface in Couette flow with a rough surface described by Cantor fractal," Heat Mass Transf, vol. 70, pp. 322-329, 2014.

[8] T. Hirabayashi, H. Takayasu, H. Miura and K. Hamada, "The behavior of a threshold model of market price in stock exchange," Fractals, vol. 1, no. 01, pp. 29-40, 1993. DOI: $10.1142 / \mathrm{S} 0218348$ X9300006X.

[9] M. Yun, B. Yu and J. Cai, "A fractal model for the starting pressure gradient for Bingham fluids in porous media," Heat Mass Transf., vol. 51, no. 5, pp. 14021408, 2008.

[10] Chen Chao, Guo Haifeng, Liu Yuning, et al. A new kind of phase change material (PCM) for energystoring wallboard," Energy and Buildings, 2008, 40, no. 5, pp. 882-890.

[11] Yinping Zhang, Kunping Lin et al. "Thermal storage and nonlinear heat-transfer characteristics of PCM wallboard," Energy and Buildings, vol. 40, pp. 1771-9, 2008.

[12] Carbonari A., de Grassi M., di Perna C., et al. "Numerical and experimental analyses of PCM containing sandwich panels for prefabricated walls," Energy and Buildings, vol. 38, no. 5, pp. 472-483, 2006. DOI:10.1016/j.enbuild.2005.08.007.

[13] Turnpenny J. R., Etheridge D. W. and Reay D. A., "Novel ventilation cooling system for reducing air conditioning in buildings part I: testing and theoretical modeling," Applied Thermal Engineering, vol. 20, no. 11, pp. 1019-1037, 2000.

[14] Uros Stritih and Vincenc Butala, "Energy saving in building with PCM cold storage," International Journal of Energy Research, vol. 31, no. 4, pp. 1532 1544, 2007.

[15] J. P. Barbour and D. C. Hittle, "Modeling phase change materials with conduction transfer functions for passive solar applications," ASME Transactions, vol. 128, pp. 58-68, 2006.

[16] Schranzhofer H., Puschnig P., Heinz A. and Streicher W., "Validation of a TRNSYS simulation model for PCM energy storage and PCM wall construction element." Ecostock Conference, 2006.

[17] Miyano Saito, Tanaka, "The variance of humidity in room according to wall materials," Architectural 
Institute of Japan, Research Report of Architectural Institute of Japan, 3rd version. Fukuoka, Shukosha Printing Co., Ltd., 1949. 21.

[18] Maddison M, Mauring T, Kirsimäe K, et al. "The humidity buffer capacity of clay-sand plaster filled with phytomass from treatment wetlands," Building and Environment, vol. 44, no. 9, pp. 1864-1868, 2009.

[19] Hasegawa T., Iwasaki S., Shibutani Y., et al., "Preparation of superior humidity - control materials from kenaf," Journal of Porous Materials, vol. 16, no. 2, pp. 129-134, 2009. DOI: 10.1007/s10934-0079176-5.

[20] Vu D. H., Wang K. S., Bac B. H., et al., "Humidity control materials prepared from diatomite and volcanic ash," Construction and Building Materials, vol. 38, pp. 1066-1072, 2013.

[21] Vu D. H., Wang K. S., Bac B. H., et al., "Humidity control porous ceramics prepared from waste and porous materials," Materials Letters, vol. 65, no. 6, pp. 940-943, 2011.

[22] Gonzalez J. C., Molina-Sabio M. and RodriguezReinoso F., "Sepiolite-based adsorbents as humidity controller," Applied Clay Science, vol. 20, no. 3, pp. 111-118, 2001.

[23] Talukdar P., Olutmayin S. O., Osanyintola O. F., et al. "An experimental data set for benchmarking 1-D, transient heat and moisture transfer models of hygroscopic building materials. Part I: Experimental facility and material property data," International Journal of Heat and Mass Transfer, 50, no. 23, pp. 4527-4539, 2007.

DOI: 10.1016/j.ijheatmasstransfer.2007.03.026.

[24] Del Coz Díaz J. J., Álvarez Rabanal F. P., García Nieto P. J., et al., "Hygrothermal properties of lightweight concrete: Experiments and numerical fitting study," Construction and Building Materials, vol. 40, pp. 543555, 2013. DOI: 10.1016/j.conbuildmat.2012.11.045.

[25] Al-Ghouti M. A., Khraisheh M. A. M., Ahmad M. N. M., et al., "Adsorption behaviour of methylene blue onto Jordanian diatomite: A kinetic study," Journal of Hazardous Materials, vol. 165, no. 1, pp. 589-598, 2009. DOI:10.1016/j.jhazmat.2008.10.018.

[26] Cachim P., Velosa A. L. and Ferraz E., "Substitution materials for sustainable concrete production in Portugal," KSCE Journal of Civil Engineering, vol. 18, no. 1, pp. 60-66, 2014. DOI: 10.1007/s12205-0140201-3

[27] Horikawa T., Kitakaze Y., Sekida T., et al. "Characteristics and humidity control capacity of activated carbon from bamboo," Bioresource Technology, vol. 101, no. 11, pp. 3964-3969, 2010. DOI: 10.1016/j.biortech.2010.01.032.

[28] Wang R. M., Wang J. F., Wang X. W., et al. "Preparation of acrylate-based copolymer emulsion and its humidity controlling mechanism in interior

wall coatings," Progress in Organic Coatings, vol. 71, no. 4, pp. 369-375, 2011. DOI: 10.1016/j.porgcoat.2011.04.007.

[29] Sungworawongpana S. and Pengprecha S., "Calcination effect of diatomite to chromate adsorption," Procedia Engineering, vol. 8, pp. 53-57, 2011. DOI: 10.1016/j.proeng.2011.03.010.

[30] Ha J. H., Oh E., Bae B., et al., "The effect of kaolin addition on the characteristics of a sintered diatomite composite support layer for potential microfiltration applications," Ceramics International, vol. 39, no. 8, pp. 8955-8962, 2013

[31] Al-Ghouti M. A., Hawari A. and Khraisheh M., "A solid - phase extractant based on microemulsion modified date pits for toxic pollutants," Journal of Environmental Management, vol. 130, pp. 80-89, 2013. DOI: 10.1016/j.jenvman.2013.08.045.

[32] Khalighi Sheshdeh R., Khosravi Nikou M. R., Badii K., et al., "Evaluation of adsorption kinetics and equilibrium for the removal of benzene by modified diatomite," Chemical Engineering \& Technology, vol. 36, no. 10, pp. 1713-1720, 2013. DOI: 10.1002/ceat.201300041.

[33] Morooka T., Homma Y. and Norimoto M., "Criterion for estimating humidity control capacity of materials in a room," Journal of Wood Science, vol. 53, no. 3, pp. 192-198, 2007. DOI:10.1007/s10086-006-0848-6

[34] Tomita Y, Takahashi R, Sato satoshi, et al., "Humidity control ability of silica with bimodal pore structures prepared from water glass," Journal of the Ceramic Society of Japan, vol. 112, no. 1309, pp. 491-495, 2004. DOI: $10.2109 /$ jcersj.112.491

[35] Watanabe O., Ishida E. H. and Maeda H., "Development of an autonomous humidity controlling building material by using mesopores," Transactions of the Materials Research Society of Japan, vol. 33, no. 2, pp. 489-492, 2008.

\section{NOMENCLATURE}

$\mathrm{C}$

W

$\mathrm{W}_{0}$

a

b

c

d

\section{Greek symbols}

$\phi$ the adsorption or desorption content of the ZBHCMs , \%

the moisture adsorption or desorption weight of the ZBHCM , kg the initial weight of the $\mathrm{ZBHCM}, \mathrm{kg}$ fitting coefficient fitting coefficient fitting coefficient fitting coefficient

ambient relative humidity thermal, $\%$ 